\title{
Experimental Study of a New Flow Control Method for Shock Train
}

\author{
Shu Sun ${ }^{1, *}$, He-xia Huang ${ }^{2}$ and Hui-jun Tan ${ }^{2}$ \\ ${ }^{1}$ College of Civil Aviation, Nanjing University of Aeronautics and Astronautics, Nanjing, China \\ ${ }^{2}$ College of Energy and Power Engineering, Nanjing University of Aeronautics and Astronautics, Nanjing, \\ China
}

\begin{abstract}
.
In order to avoid violent stream-wise and transverse oscillation in isolator, a fluidic control method was proposed. This method combines the boundary suction and the slot injection method together. For the sake of checking out the control effect of the concept, two contrast wind-tunnel experiments were completed in $\mathrm{M} 0=4.92$ hypersonic wind tunnel. According to the experiment results, as the shock train moving upstream, the mass flow rate of secondary flow increased and the slip line moving towards to the bottom wall, meanwhile a series of weak shocks generated by injection got stronger that increased the pressure upstream the shock train. The shock train interacted with upstream background shockwaves, and the travelling processes can be divided into 4 stages which were much sim-pler than that of uncontrolled flow. Although there was one unstable stage, the shock train behaved in a much milder manner with an oscillation frequency of $641 \mathrm{~Hz}$ and the maximum oscillation power decayed to $20 \%$ of that of uncontrolled flow, and the maximum sustainable back pressure almost equal to that of uncontrolled flow.
\end{abstract}

Keywords: shock train, flow control, wind tunnel experiment, hypersonic inlet, isolator

\section{Introduction}

Shock train is a special flow phenomenon which results from shock/boundary layer interaction that is frequently encountered in many fluid devices such as hypersonic inlet/isolator, supersonic wind tunnel diffusers ${ }^{0}$ etc. The flow structure in shock train region is very complicated, and the air in the region would experience the circulative process of compression>expansion->re-compression. However, the shock train is unavoidable in hypersonic inlet/isolator due to the following reasons. Firstly, shock train is a crucial factor that determines whether the engine can operate in stable-state ${ }^{0}$, once the combustor is over fueled, the shock train may be disgorged out of isolator, which results in the inlet unstart. Secondly, the performance of inlet/isolator is also determined by the strength of shock train and its relative position in isolator ${ }^{0}$. Finally, shock train serves as a pre-combustion shock when the dual mode scramjet works in the ramjet mode ${ }^{0}$. In order to take the advantages of shock train to reach a maximum pressure rise with less total pressure loss and suppress the flow oscillation, several 
control methods have been developed, which can mainly divided into passive control and active control.

For passive control method, the main characteristic is the control variable can't change with the flow state, mainly included: vortex generator ${ }^{0}$, the mechanism of this technique is using vortex generator to energize the near wall low-energy flow, another passive control method is passive porous cavity ${ }^{0}$, with a porous plate to separate the cavity flow from the core flow, which generate a series of weak shock waves to increase upstream pressure of shock train, can also improve the resistance ability to high back pressure. While the active method is more flexible, the control effect alters as the flow states changing, mainly include boundary layer suction ${ }^{0-0}$, which can stabilize the primary foot of shock train and increase the back pressure to almost normal shock pressure rise with the expense of mass lost about $24 \%$ mass flow rate of mainstream. In contrast, slot injection method injects a mass of high-energy secondary flow into the core flow, which can improve the normal momentum of the core flow, and suppress the self-excited oscillation phenomena ${ }^{0}$ etc. So to improve the boundary layer kinetic is the key factor for shock train control method. However, the previous research mainly focus on the characteristics of shock train on direct-connect facilities ${ }^{0-0}$, and relatively less work have been done on isolator with the influence of inlet background waves ${ }^{0-0}$.More less work on the shock train control method under the integrated inlet/isolator condition can be seen. According to our previous research, shock train oscillates substantially in hypersonic inlet/isolator ${ }^{0}$, which may lead to flameout. So an effective control method to restrain shock train oscillation in integrated inlet-isolator model is quite necessary.

The motivation for the work presented in this paper is derived from the idea to combine the boundary layer suction under shock train with the slot injection method together to integrate the superiority and avoid the disadvantage of these two methods. In this paper, a selfcirculate fluidic control method in hypersonic inlet/isolator model is proposed. And several wind-tunnel experiments with and without this control method have been conducted to examine the control effect according to the high frequency pressure sensor signals and high speed Schlieren pictures.

\section{Description of the Fluidic Control Method Concept}

Fig 1-Fig 2 show the shock train zone in the isolator, it can be seen from Fig 3 that the pressure in shock train zone is significantly higher than that of upstream flow. To utilize the differential pressure, a secondary flowpath was designed from shock train zone to upstream. The control method is schematically shown in Fig 4. A fraction of secondary flow driven by the differential pressure is introduced to the upstream primary flowpath smoothly through a multi-slot plate and the chamber. A valve is used in the chamber to keep the mass flow rate of secondary flow at an appropriate value. For the forepart, the secondary flow act as slotinjection due to the displacement effect and normal momentum injection which generates a series of weak shock waves that increase the upstream pressure and impulse of shock train. For the rear part, especially in the shock train zone, the low-energy flow under shock train is removed continually like boundary layer suction which set barriers to the shock train can stabilize the primary shock foot.

Compared to the traditional active control method, the distinctive characteristics of this fluidic control method is zero mass lost and no additional external fluid sources, and the secondary flow is circulative using with the amount about $1 \%$ capture mass flow rate of the inlet. 
The controlled object of this control method is quite simple, just need to control the opening of the valve that settled in the pressure stabilizing cavity. Moreover, there is no additional block in the inlet which has no effect on the inlet starting characteristics compared to the passive control method.

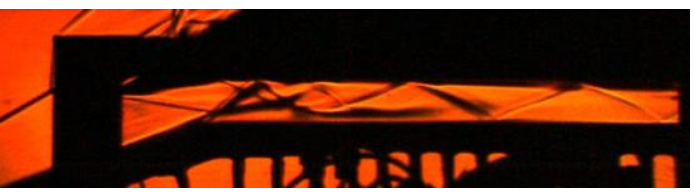

Fig 1. $M 0=4.92$, unthroattled Flow $(A R=1.125)$

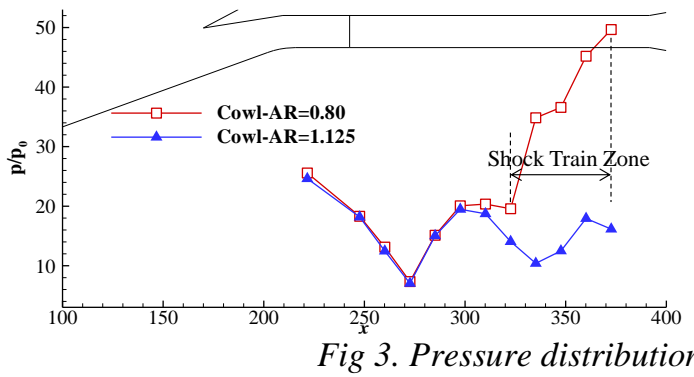

Fig 3. Pressure distribution characteristic in shock train zone

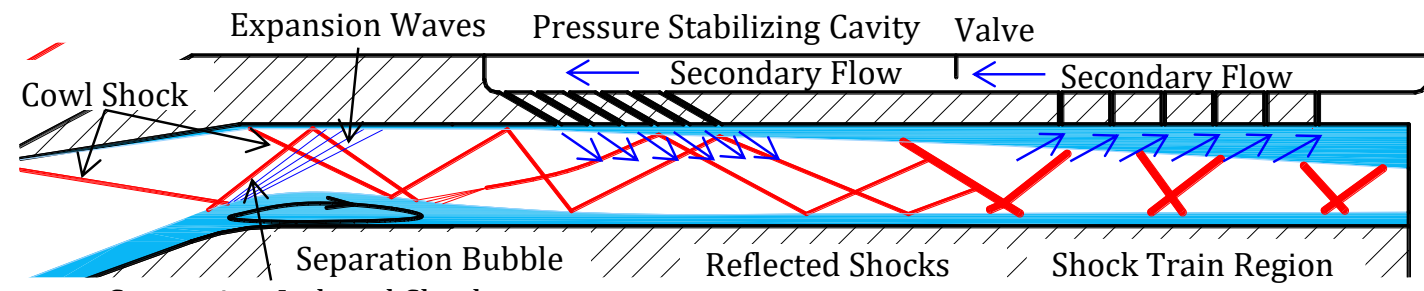

Separation-Induced Shock

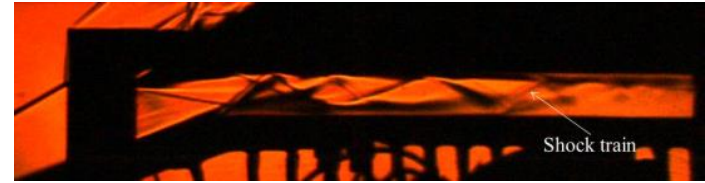

Fig 2. $M 0=4.92$, with shock train in the isolator $(A R=0.80)$

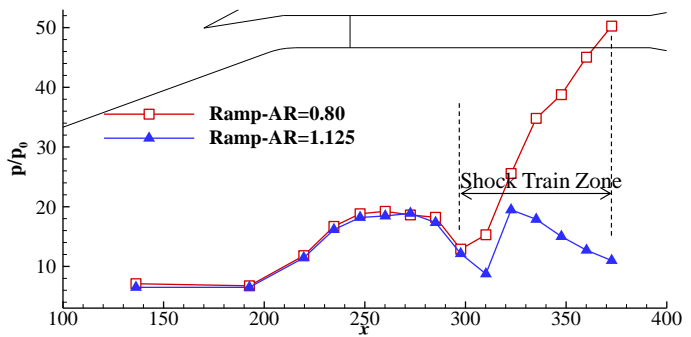

Fig 4. The Concept of Fluidic Control Method of Shock Train in Hypersonic Inlet/Isolator

\section{Experimental facilities and Test Model}

In order to verify the control concept, several experiments with incoming Mach number 4.92 had been conducted at $\Phi 500 \mathrm{~mm}$ hypersonic wind tunnel (NHW) in Nanjing University of Aeronautics and Astronautics. The NHW is a blow-down type wind tunnel with an electric metal storage heater installed upstream the pressure chamber. The facility can work at nominal Mach number range from 4.0-8.0 and flight conditions from 27 to $59 \mathrm{~km}$ with usable time over 7s.For current experiments, the Mach number 5.0 nozzle was used, and the freestream condition was listed below.

\begin{tabular}{ll} 
Table 1. The Freestream condition for the experiments \\
\hline Nominal mach number & 5.0 \\
Actual Mach Number & 4.92 \\
Freestream static pressure $(\mathrm{Pa})$ & 1928.22 \\
Freestream Static Temperature $(\mathrm{K})$ & 89.99 \\
Total Temperature $(\mathrm{K})$ & 525.67 \\
\hline
\end{tabular}

The test model is a typical two-dimensional hypersonic inlet/isolator, which is shown in Fig 5. The ramp angle of the inlet is 20 degree to the freestream flow direction and the corresponding deflection angles of the two cowl shocks are 11 degree and 9 degree respectively. In order to make a comparison between the uncontrolled flow with the controlled flow, the control units were designed replaceable. Fig 6 shows the main parameters of the model, Where 
$H_{\text {iso }}$ represents the height of throat $\left(H_{\text {iso }}=16 \mathrm{~mm}\right)$. For the model with controlled units, twelve slots were used with 6 injection slots and 6 suction slots; the width $W_{\mathrm{s}}$ of all the slots is $1 \mathrm{~mm}$. The injection slots are perpendicular to the core flow, while the included angle between the injection slots and the core flow is $27 \mathrm{deg}$.In the current model, the mach number of the entrance $\mathrm{M}_{\mathrm{in}}=2.98$ corresponding to the incoming mach number of 4.92 .

In order to give a better view of the internal flow-field, two pieces of transparent quartz glass were set at both side of the isolator. A Toepler z-type Schlieren system is used, all Schlieren images shown are recorded by a MotionExtra LG-100K high speed camera from RedLake, Inc. The sampling rate of the Schlieren visualization is set at 1000 frames per second. A flow plug driven by a linear electric motor was assembled at the exit of the isolator to simulate combustion-induced high pressure. In addition, 28 pressure sensors with the sampling frequency $20 \mathrm{KHz}$ were equipped in the top and bottom walls of the isolator so as to give more details of the highly unsteady flow-field. For the model with controlled units only 15 pressure sensors installed on the bottom wall were used. The pressure data were acquired by National-Instruments DAQ USB-6255 Data Acquisition Card. In order to keep the Schlieren pictures synchronized with the pressure signal, a pulse signal was used to trigger the camera; meanwhile, the pulse signal was gathered by the DAQ system.

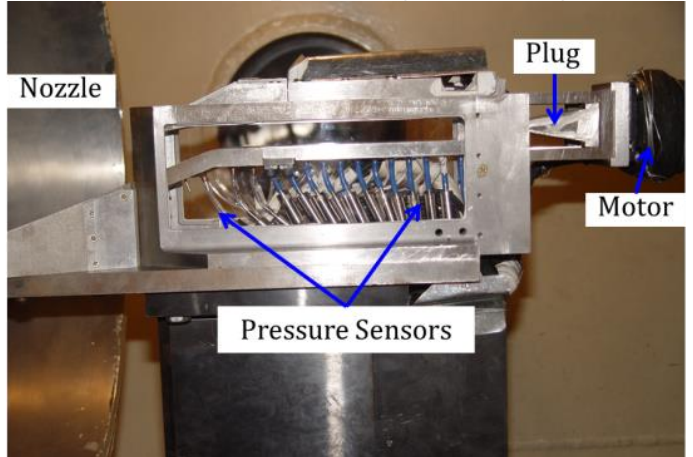

a.Basic Model(Uncontrolled)

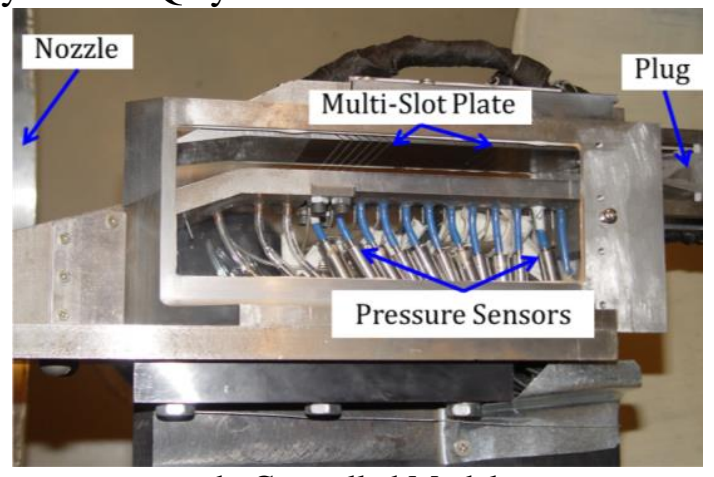

b. Controlled Model

Fig 5. Test model installed in the hypersonic wind tunnel

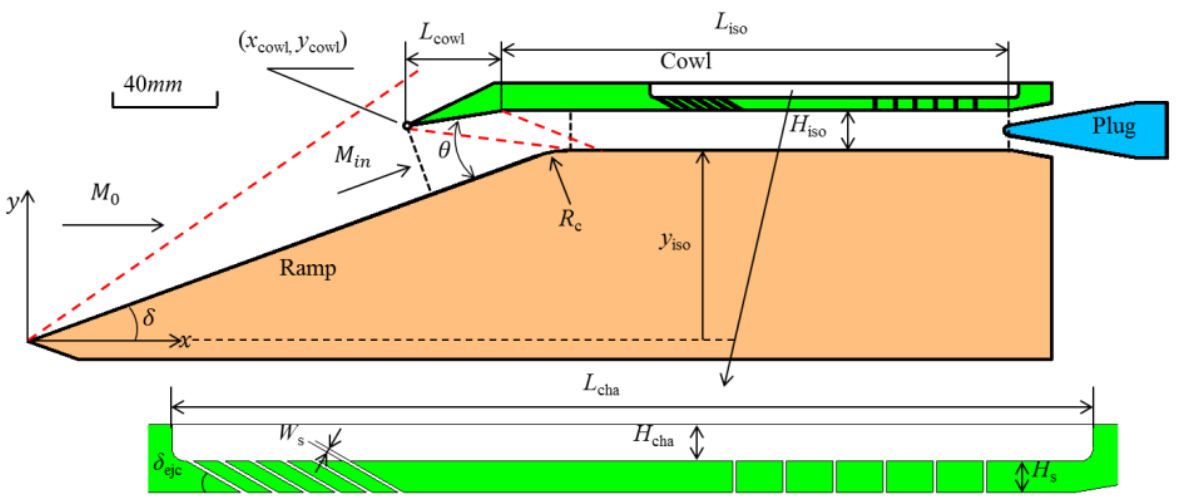

Fig 6. Sketch of the hypersonic inlet/isolator

\section{Results and Discussion}

\subsection{Unthrottled Flow Field}

The plug were set at the most downstream position initially which doesn't form effective block, the inlet/isolator were at unthroattled state. Fig 7 shows the unthroattled flow structure 
of the uncontrolled flow and the controlled flow. For the uncontrolled flow, the two cowl shocks induce the boundary layer to separate, a large-scale separation bubble can be seen from the Schlieren picture. Due to the separation, some additional shocks were formed, mainly including the separation-induced shock and the reattachment shock, moreover, the original cowl shock reflection process was damaged by the separation, the reattachment shock reflected in the isolator about 4 times which constituted the primary shocks of the isolator. The controlled flow structure upstream the injection slots were same to the uncontrolled flow. Although there's no shock train in the isolator at unthroattled state, a slip line can be seen from the Schlieren picture, which means that a fraction of secondary flow were introduced to the core flow mainly due to the fact that the downstream pressure is also higher than upstream with a quite small driven pressure. The slip line separated the relative low-energy secondary flow from the core flow, was about $0.15 \mathrm{H}_{\text {iso }}$ to the top surface of the isolator. Meanwhile, several weak shocks were generated near the slots; these shocks interacted with the reattachment shock and reflected in the isolator together. The reflection process of the reattachment shock was quite different from the uncontrolled flow, it reflected on the bottom wall as well as the slip line and become very weak so that it almost couldn't be seen from the Schlieren figure at the rear part of the isolator.
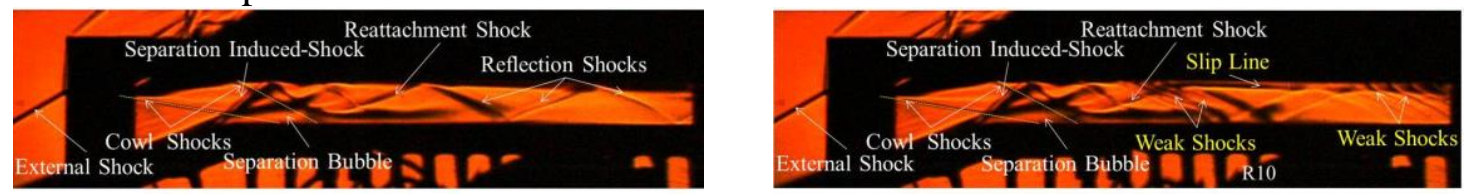

Fig 7. Unthroattled flow structure comparison

For the reason that there are no pressure sensors on the cowl side of the controlled model, just the static pressure on the ramp surface was measured. Fig 8 gives the pressure distribution normalized by the incoming static pressure $\mathrm{p}_{0}$; it's obvious that the pressure distribution before measure point R10 was almost the same (the measure point R10 was marked in Fig 7). A series of shocks were behind of R09 resulting a pressure arising of R10 compared to the uncontrolled flow. For the uncontrolled flow, there's only reattachment shock and expansion wave reflected, the pressure distribution showed a wavelike shape, the wave trough was the place where the reflection points lied like point R10, the pressure rising from R10 to R11 were resulting from the reflection shocks, and during the point R11 to R15 were the expansion area. For the controlled flow, there were two reflection points (R10, R13) which represented the reflection point of the weak shocks and the reattachment shock respectively and the pressure rising by the reflection shocks was quite smaller that meant the shocks became weaker compared to the uncontrolled flow.

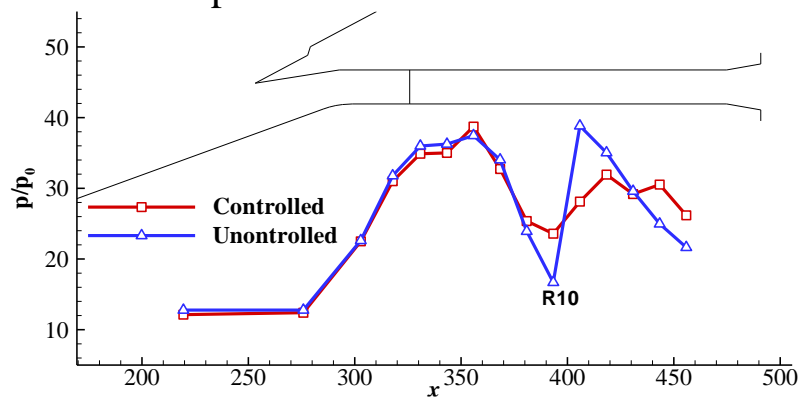

Fig 8. Pressure distribution on the ramp between the Controlled and the uncontrolled flow 


\subsection{Stage1, the shock train crossed over the suction slots}

Fig 9 displays the shock train crossing over the six suction slots process. At $t=5094 \mathrm{~ms}$, shock train began to appear in the visual field and the top branch of the shock train reached the $6^{\text {th }}$ suction slots. As the plug moving upstream, shock train continued to moving upstream and reached the remaining suction slots at $5438 \mathrm{~ms}, 5660 \mathrm{~ms}, 5900 \mathrm{~ms}, 6141 \mathrm{~ms}$ and $6394 \mathrm{~ms}$ respectively. During stage 1 , the core flow continuously deflected to the top wall until $\mathrm{t}=6394 \mathrm{~ms}$, then the core flow switched to deflection to the bottom wall. Compared to the uncontrolled flow field, the shock train was so obscure, it hardly to distinguish the shock train from the weak shocks. And the shock train kept at a more downstream position with same $\mathrm{AR}$, comparing to the uncontrolled flow. For $\mathrm{AR}=0.801$, the leading edge of the shock train arrived at the middle of R13 and R14, while the leading edge of shock train reached the middle of R12 and R13 for uncontrolled flow. Meanwhile, From Fig 10, it can be seen that the pressure in the shock train zone increased leading to a rising of the secondary mass flow rate. The distance of the slip line to the top surface of the isolator increased from about $0.15 \mathrm{H}_{\text {iso }}$ to $0.26 \mathrm{H}_{\text {iso }}$ resulting the upstream flow reconstruction. It can be seen from Fig 10 that the pressure of upstream measurement points R08-R11 were changed as the shock train moving, which shows a difference from the uncontrolled flow. What's more, Fig 11 gives the pressure-time histories of R08-R10. For R08, the pressure rising significantly during $t=5094 \mathrm{~ms}$ to $\mathrm{t}=5438 \mathrm{~ms}$, the pressure of R09 mainly increasing at $\mathrm{t}=5094 \mathrm{~ms}$, while the pressure of R10 increasing all the time during Stage 1. These measure points were all under the influence of the injection weak shocks, the difference was that each measure point was influenced by different weak shocks according to their positions. The difference between R08-R10 meant that the weak shocks generated near the different injection slots changed different, the more downstream the slots located the more significant influence on the shocks. On the other hand, as the shock train reaching one slot, the weak shock generated near the other upstream suction slots became more and more clear, which meant the weak shocks turned to be stronger, that was, the remaining suction slots acted as injection slots, the secondary flow ejected to the core flow not only through the injection slots but also through the upstream suction slots.
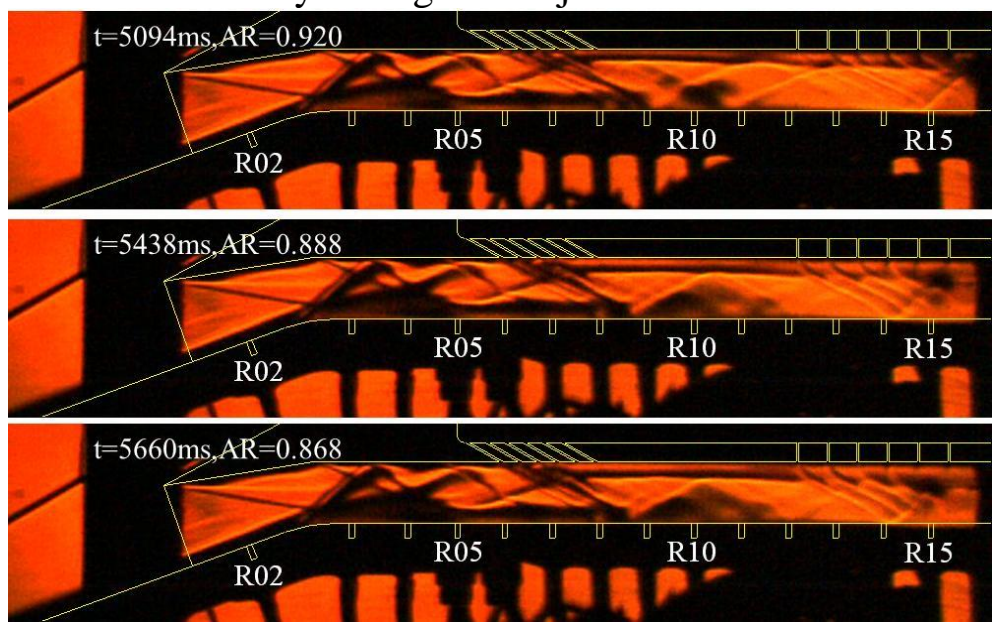

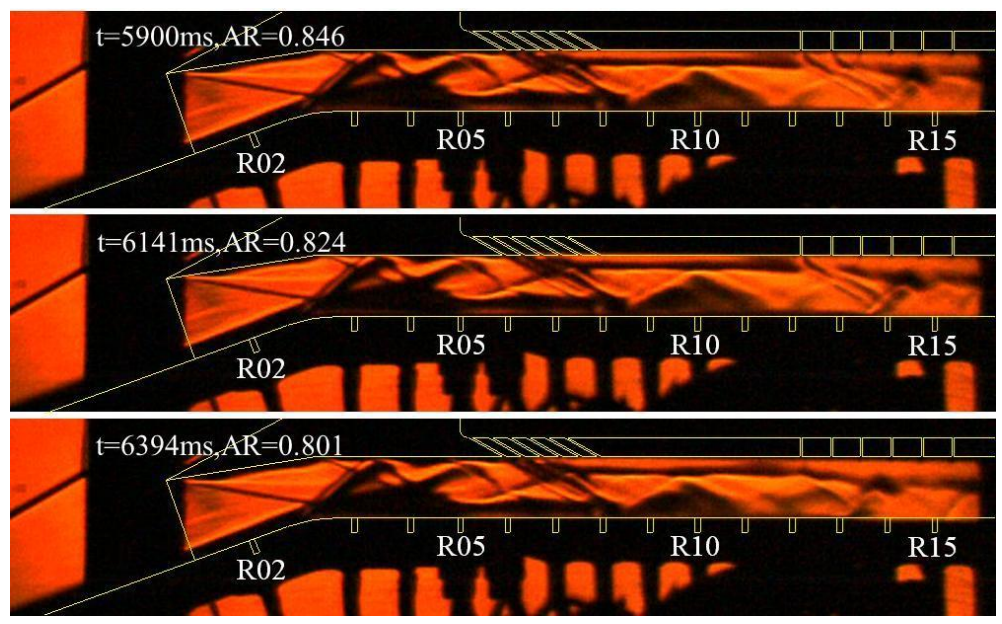

Fig 9. Schlieren pictures during stage 1

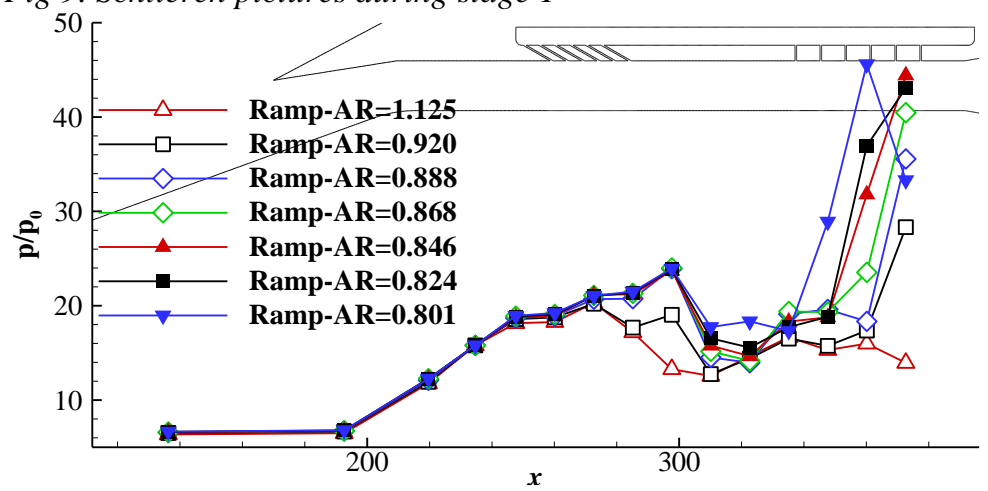

Fig 10. Pressure distribution on the ramp during stage 1

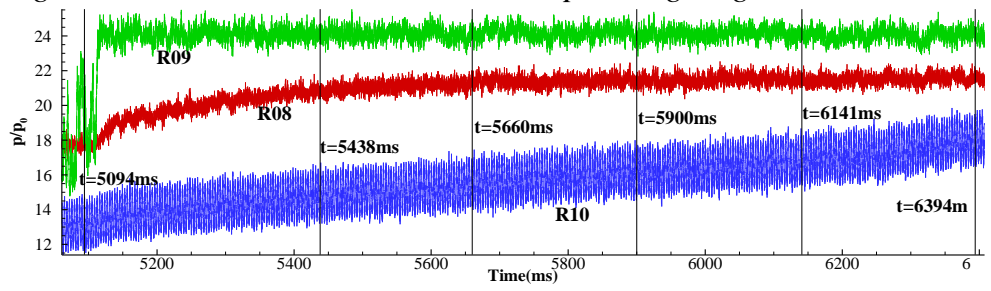

Fig 11 .Typical pressure measurement points pressure-time histories during stage 1

\subsection{Stage 2, secondary mass flow rate increasing significantly}

After stage 1, the weak shocks of suction slots were destroyed and the core flow deflected to the bottom wall, all the suction slots were sunk into large scale separation and low-energy fluids, and the separation under shock train on the top wall was connected with the injected secondary flow. As AR decreased from 0.795 to 0.776 , the slip line moved down slowly. For $\mathrm{AR}=0.795$, the distance between slip line and the top wall was about $0.27 \mathrm{H}_{\text {iso, }}$, while the distance increased to $0.33 \mathrm{H}_{\text {iso }}$ when $\mathrm{AR}=0.776$. When $\mathrm{AR}$ decreased to 0.769 , the slip line moving down a lot and the separation near the injection slots increase obviously, the weak shocks generated by the injection slots were also destroyed and the flow in the isolator rebuilt, the leading edge of shock train moving to the injection slots. The leading shock swept over R08 resulting an enormous pressure rise. During this stage, the core flow deflected to the bottom wall leading to a wavelike pressure distribution on the ramp. The flow characteristics at this state were very similar to the stage 5 of uncontrolled flow; the difference was that stage 2 of 
controlled flow lasted a long time about $353 \mathrm{~ms}$ with a relative large AR, while stage 5 of uncontrolled flow just lasted less than $20 \mathrm{~ms}$.

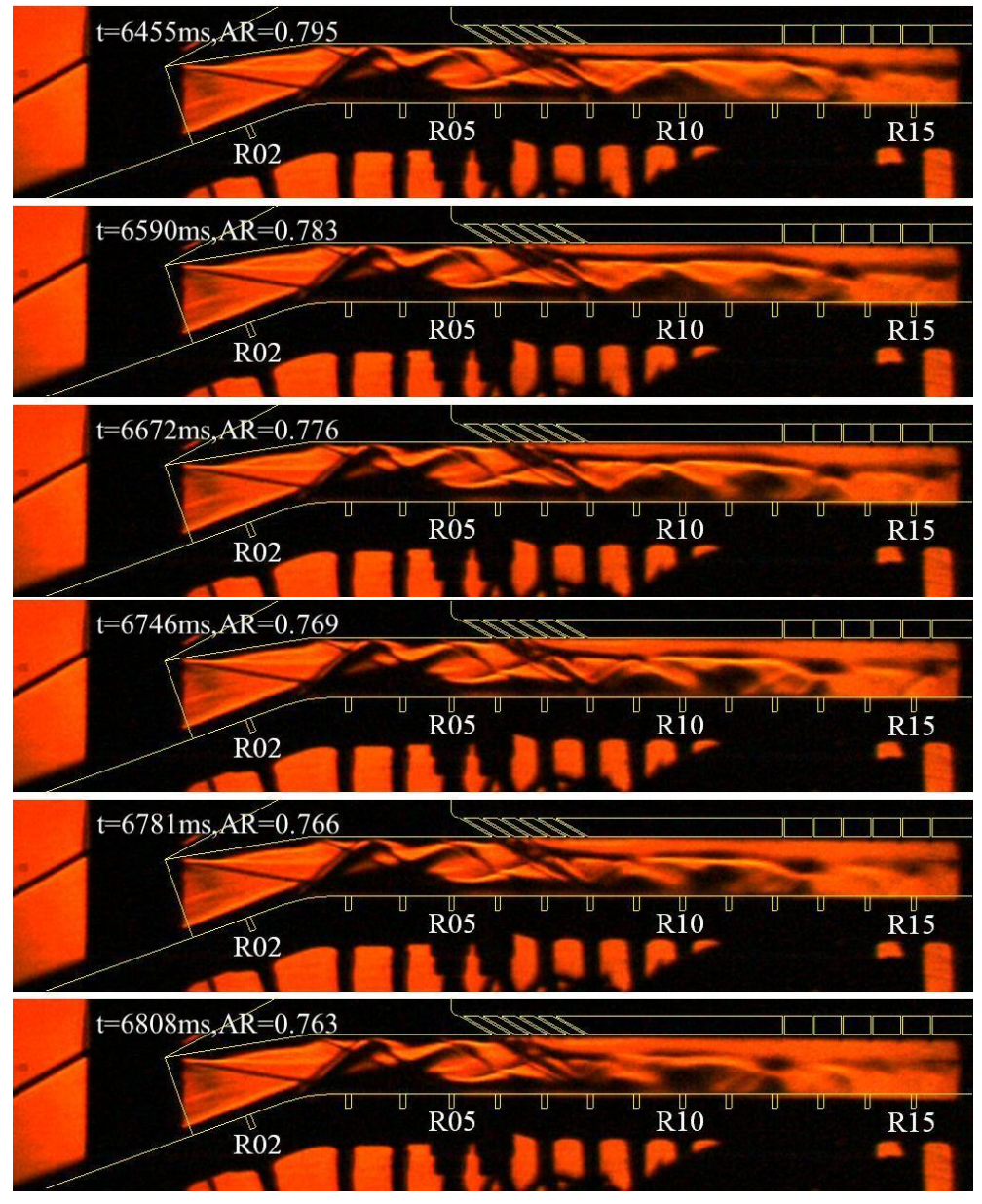

Fig 12. Schlieren pictures during stage 2

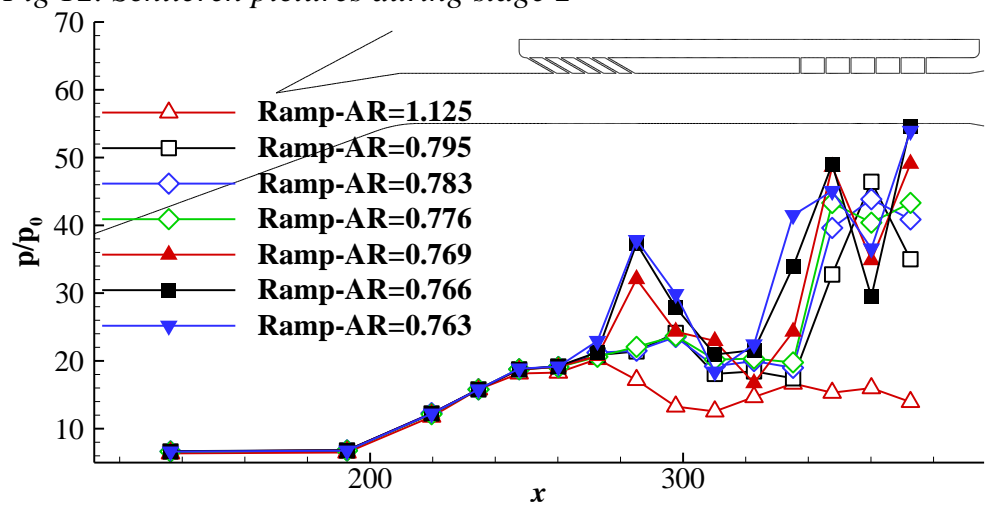

Fig 13. Pressure distribution on the ramp surface during stage 2

\subsection{Stage 3, high frequency self-oscillation observed}

After the stable state of stage 1,2, the shock train intersected with the reattachment shock, very similar to the stage 6 of uncontrolled flow. Fig 14 shows two typical oscillation cycles 
during stage 3. Compared to the uncontrolled flow, the shock train oscillated in a much milder manner. It can be seen from Fig 14 that the core flow kept deflect to the bottom wall, one can also see that the oscillation didn't create violent stream-wise and transverse swing, it's so hard to distinguish the position changing of the leading shock from Schlieren pictures that the leading shock of the shock train varying in a tiny distance. at $t=6858 \mathrm{~ms}$ the top branch of the shock train arrived at the second injection slots, at $\mathrm{t}=6859 \mathrm{~ms}$, the separation bubble under shock train increased, shock train moved upstream and merged with the weak shock generated by the first injection slot, then the shock train began to moving downstream, an oscillation cycle were built. According to the pressure signal of R08, the frequency of oscillation was $641 \mathrm{~Hz}$ and the power spectral density of R08 was $8.28 \mathrm{kpa}^{2}$, which was about $20 \%$ of the uncontrolled flow. To be honest, the frequency of the high speed camera was relative low that it's hard to catch the flow changing during this high frequency oscillation. Fortunately, the sampling frequency of the DAQ system was enough to record the highly unsteady pressure
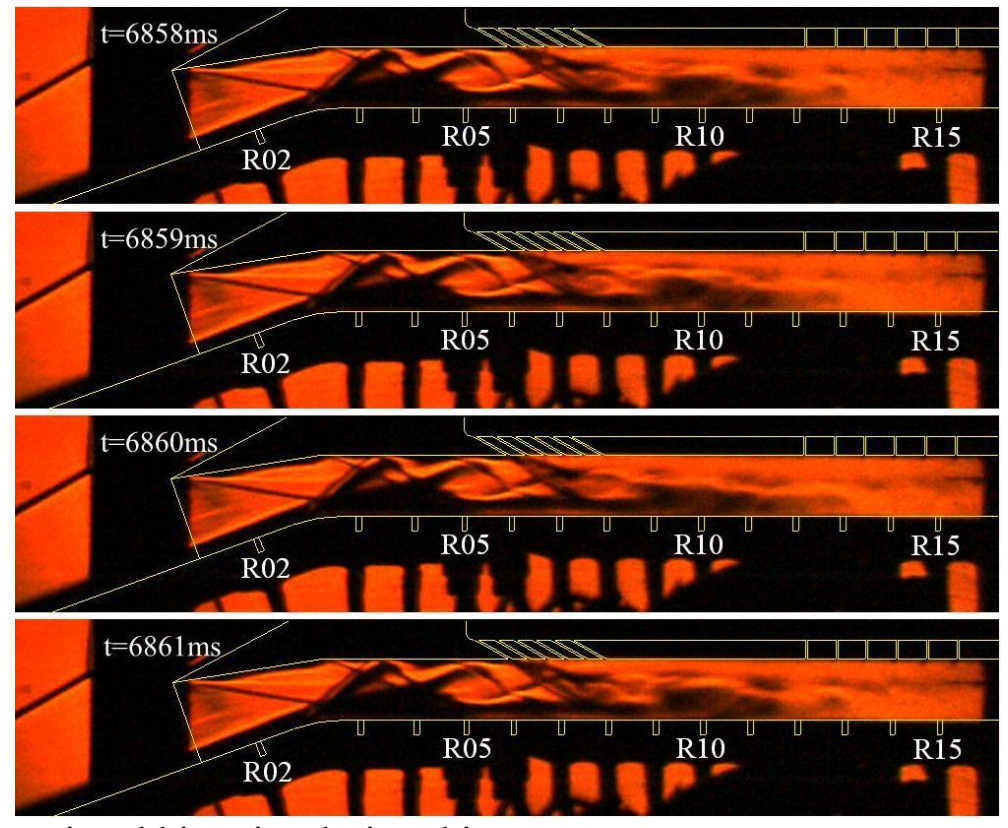

signal histories during this stage.

Fig 14. Schlieren pictures during stage 3
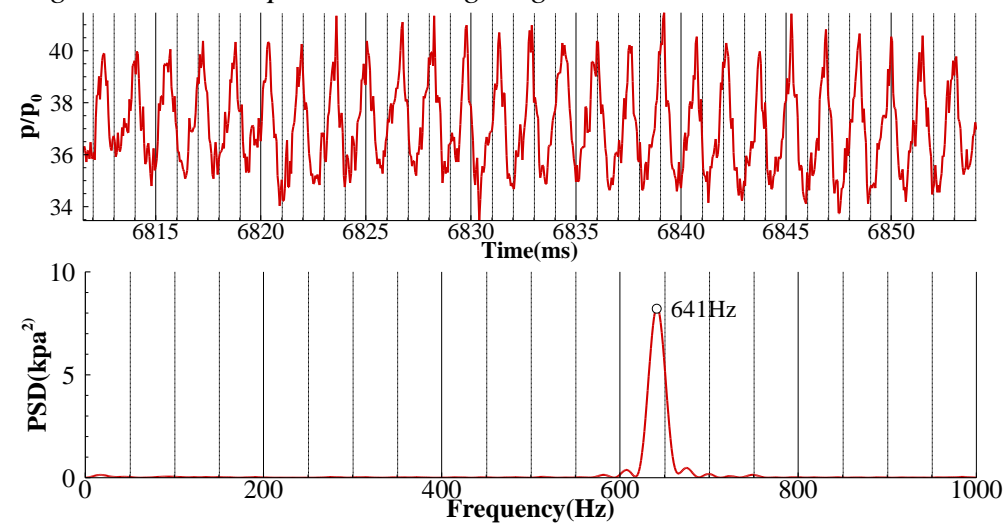

Fig 15. Pressure PSD features of typical measuremnet point 


\subsection{Stage 4, the shock train interacted with the reattachment shock}

After stage 3, the shock train intersected with the reattachment shock, a relative stable state was achieved again. During this stage, the core flow continuously deflected to the bottom surface, and all the slots were surrounded by the low-energy fluid. It can be seen from Fig 16 that the low-energy fluid moved towards to the bottom wall as the plug moving upstream and the pressure in the shock train zone increasing continuously (Fig 17), which were quite similar to stage 7 of uncontrolled flow. The difference between these two stages were that the ARs of the controlled flow were relative larger than that of the uncontrolled flow, in other words, the inlet/isolator with the controlled method were more easily to realize this flow state.
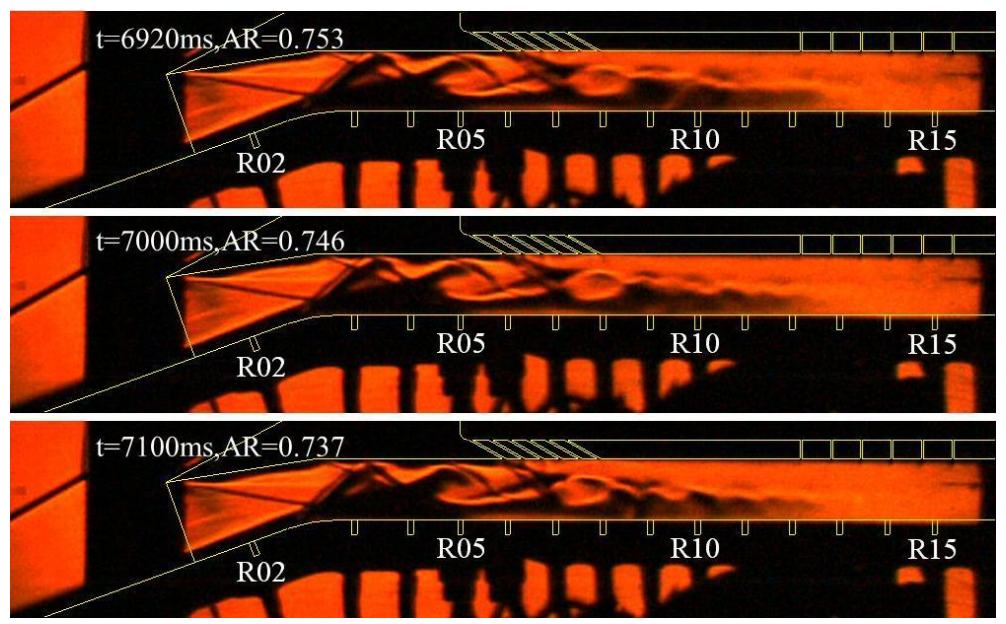

Fig 16. Schlieren pictures during stage4

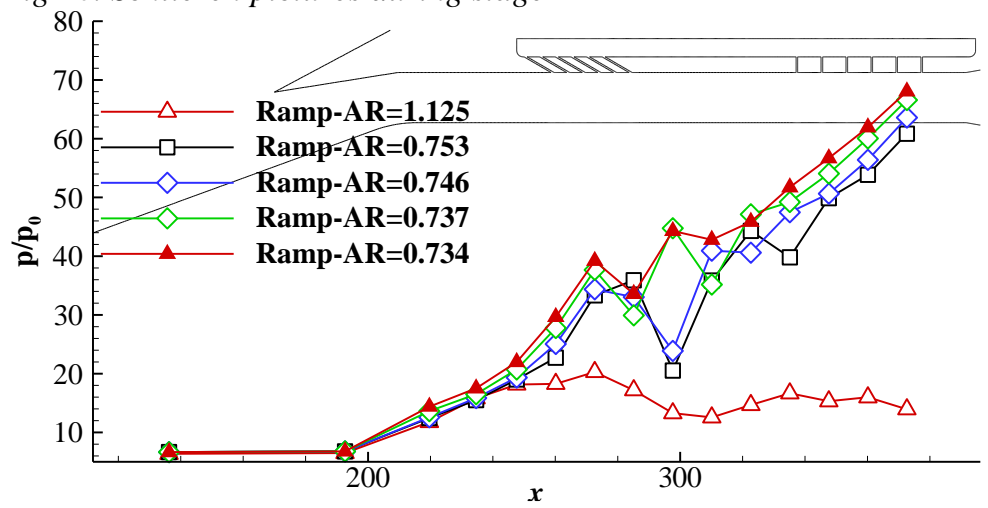

Fig 17. Pressure distribution on the ramp surface during stage 4

After the short stable state, the inlet/isolator fell into "little buzz" state at $t=7143 \mathrm{~ms}$ with an oscillation frequency of $641 \mathrm{~Hz}$ that was same to the frequency of stage 3 , while the frequency was lower than that of the uncontrolled flow, mainly because of the volume of the isolator was much larger. At $\mathrm{t}=7250 \mathrm{~ms}$, the fluid in the isolator stopped to oscillate and reached the maximum sustainable back pressure of $76 \mathrm{p}_{0}$ which was a little less than the uncontrolled flow. 


\section{Conclusion}

Two wind-tunnel experiment models without and with controlling units were designed and two wind-tunnel experiments have been conducted at $\mathrm{M} 0=4.92$ hypersonic wind tunnel. The detailed characteristics of shock train in isolator were compared. The experiments shown that the travelling process of shock train without controlled flow could be divided into seven typical stages according to which shock was the shock train interacted with. And four stable stages were found in these seven stages. The shock train switched from one stable stage to another have to experienced one unstable stage. During the unstable stage, violent stream-wise and transverse oscillation occurred. The more upstream the shock train located the lower of oscillation frequency was got. When the shock train switched from intersecting with the first reflection shock of reattachment shock to the reattachment shock, the oscillation frequency reduced to $72 \mathrm{~Hz}$ with the maximum oscillation power up to $41 \mathrm{Kpa}^{2}$. For uncontrolled flow, the maximum sustainable backpressure was 78 times of incoming static pressure.

Due to the differential pressure between upstream and downstream, a fraction of secondary flow sucked out to the chamber and injected to the core flow, a series of weak shocks were generated by the slots, and a slip line can be seen that separated the secondary flow from the core flow, the slip line as well as these weak shocks changed the background shocks upstream the shock train, the travelling process of shock train in controlled isolator became much simpler, four stages including only one unstable stage were found. As the shock train moving upstream, the mass flow rate of the secondary flow increased leading to the weak shocks become stronger that increased upstream pressure as well. During the unstable state, the leading shock of shock train oscillated in a much milder manner, the position of the leading shock almost not changed, moreover the oscillation frequency increased to $641 \mathrm{~Hz}$ while the maximum oscillation power reduced to $20 \%$ of that of controlled model. The oscillation process was effectively suppressed at the expense of not significantly weaken the ability to bear the back pressure. In conclusion, this fluidic control method is useful to control shock train in hypersonic inlet/isolator.

\section{Acknowledgment}

This work is funded by the National Natural Science Foundation of China (grant nos. 51906104, 12025202, U20A2070, and 11772156), the Natural Science Foundation of Jiangsu province (grant no. BK20190385), the priority academic program development of Jiangsu higher education institutions, and the Fundamental Research Funds for the Central Universities (grant nos. 1002-YAH18026 and 1002-56XAA19050).

\section{References}

Kazuyasu Matsuo, Yoshiaki Miyazato, Heuy-Dong Kim. Shock train and pseudo-shock phenomena in internal gas flows. Progress in Aerospace Sciences .Vol. 35.1999,pp. 33-100

Hui-jun Tan,Liu-gang Li,Yu-fen Wen,Qi-fan Zhang. Experimental Investigation of the unstart process of a genetic hypersonic inlet. AIAA Journal.Vol.49,No.2,Feb, 2011,pp. 279288

Hui-jun Tan, Shu Sun. Preliminary study of shock train in a curved variable-section diffuser. Journal of Propulsion and Power,Vol.24,No.2,March,2008,pp.245-252 
Curran ET, Heiser WH, Pratt DT. Fluid phenomena in scramjet combustion systems. Annu Rev Fluid Mech .Vol.28, Jan, 1996. pp. 323-360.

McCormick DC. Shock/boundary-layer interaction control with vortex generators and passive cavity. AIAA Journal.Vol.31,No.1, 1993, pp.91-96.

Weise A. The separation of flow due to compressibility shock. NACA TM No.1152, 1947.

Wong WF. The application of boundary layer suction to suppress strong shock-induces separation in supersonic inlets. AIAA Paper 74-1063. 1974.

Weiss ,H. Olivier. Behavior of a shock train under the influence of boundary-layer suction by a normal slot. Exp Fluids, Vol. 52, 2012, pp.273-287.

Hidetaka OKUI, Hisashi HIRAKI, et al. Control of Pseudo-Shock by Slot Injection. JSME International Journal. Series B, Vol. 45, No.1, 2002.pp.150-157.

P. J. Watrup , F. S. Billig. Prediction of Precombustion Wall Pressure Distributions in Scramjet Engines. Journal of Spacecraft. Vol. 10, No.9.1976.pp.620-622.

G. Sullins, G. McLafferty. Experimental Results of Shock Trains in Rectangular Ducts. AI$A A-92-5103.1992$

John R. Hutzel, Douglas D. Decker. Scramjet Isolator Shock-Train Leading-Edge Location Modeling. AIAA 2011-2223.2011

Matthew L. Fotia, James F. Driscoll. Isolator-Combustor Interactions in a Direct-Connect Ramjet Scramjet Experiment. Journal of Propulsion and Power. Vol. 28, No. 1. Jan, 2012.pp.83-95.

Birgit U. Reinartz, Carsten D. Herrmann, Josef Ballmann.et al. Analysis of hypersonic inlet flows with internal compression. AIAA 2002-5230.2002

B.U. Reinartz, C.D. Herrmann, Josef Ballmann, W. Koschel. Aerodynamic Performance Analysis of a Hypersonic Inlet Isolator Using Computation and Experiment. Journal of Propulsion and Power. Vol. 19, No. 5, Sep. 2003.pp.868-875.

Zhang Kunyuan, Wang Chengpeng, Yang Jianjun, Xu Jinglei. Investigation of flow in isolator of hypersonic inlet. Journal of Propulsion Technology. Vo1.23,No.4.2002,(In Chinese)

H.J. Tan, S. Sun, H.X. Huang. Behavior of Shock Trains in a Hypersonic Inlet/Isolator Model with Complex Background Waves. Exp Fluids. Vol.53,No.6.Sep.2012.pp.1647-1661 